\title{
Nonsingular gradient solutions in crack mechanics and the concept of stress concentration.
}

\author{
Valery Vasiliev \\ Department Mechanics of Strength and \\ Fracture of Materials and Structures \\ Ishlinsky Instituite for Problem in \\ Mechaanics of Russian Acad. of Scs. \\ Moscow, Russia
}

\author{
Sergey Lurie \\ Department of Nonclasiscal Models of \\ Composite Materials \\ Instituite of Applied Mechanics of \\ Russian Acad. of Scs \\ Moscow, Russia
}

\author{
Dmitrii Volkov-Bogorodskii \\ Department of Nonclasiscal Models of \\ Composite Materials \\ Instituite of Applied Mechanics of \\ Russian Acad. of Scs \\ Moscow, Russia
}

\begin{abstract}
We consider the fracture mechanics problem for the finite and semi-infinite cracks in the gradient elasticity. Local stress fields that define the fracture the strength of materials are found as solutions of the inhomogeneous Helmholtz equations in which the inhomogeneity is determined by classical stresses. To construct solutions, the radial factors method and the Papkovich-Neuber representation are used. It is shown that, in problems of crack mechanics. We show that the local stresses in the vicinity of crack tips are non-singular, have the form characteristic of stress concentration, and depend only on the level of acting stresses and the scale parameter, whicl $\backslash$ is found as a result of mechanical testing of material samples.
\end{abstract}

Keywords-singular problems; gradient elasticity; complex potentials; radial multipliers, regular gradient solutions

\section{INTRODUCTION (HEADING 1)}

In recent years, the problem of singularities in the problems of elasticity theory and crack mechanics has been widely discussed [1,2]. The singularity of solutions for stresses in the linear theory of elasticity at the crack tip excludes the application of criteria for the strength of bodies with stress concentration. On the contrary, gradient elasticity provides regularization of singular solutions of differential equations of the theory of elasticity [3-6], allows one to describe dimensional effects and allows the possibility of using traditional approaches for the assessment of strength. As a short introduction to nonlocal elasticity, we present the following simple considerations. Let's consider a plane problem and introduce a generalized function $U(x, y)$ which is equal to the average value of the function $u(x, y)$ in a rectangular representative fragment $-a / 2 \leq \alpha, \beta \leq a / 2$

$$
U(x, y)=\left(1 / a^{2}\right) \int_{-a / 2}^{a / 2} \int_{-a / 2}^{a / 2} K(\alpha, \beta)(u(x, y ; \alpha, \beta) d \alpha d \beta
$$

where $K(\alpha, \beta)$ - is the core of averaging

Next, we use the expansion of the integrand in a power series in local coordinates

$$
\begin{aligned}
& u(x, y ; \alpha, \beta)=u(x, y)+\alpha u_{, x}+\beta u_{, y}+ \\
& +(1 / 2 !)\left(\alpha^{2} u_{, x x}+2 \alpha \beta u_{, x y}+\beta^{2} u_{, y y}\right)+\ldots
\end{aligned}
$$

It can be shown that there exists such an averaging core that, after substituting (2) into (1) and integrating over the fragment, we obtain $U(x, y) \approx u(x, y)-s^{2} \Delta u(x, y)$, where $s$ is the scale parameter. Nonlocal functions can also be introduced for vector and tensor fields that determine the stress-strain state of a plane problem. In particular, it is shown that the equilibrium equations can be rewritten in terms of generalized stresses [5,6] $\tau_{i j}: \tau_{i j}=\left(\sigma_{i j}-l^{2} \Delta \sigma_{i j}\right)$. It is believed that Hooke's laws are formulated in terms of generalized stresses and generalized deformations, which is natural from a physical point of view. Local stresses are used to assess strength. During the solution of the problem in stresses, the first stage considers the traditional boundaryvalue problem for generalized stresses $\tau_{i j}$, and hence also for generalized deformations and displacements. At the second stage, the obtained function. At the second stage, the obtained function $\tau_{i j}$ is substituted into the equation $\tau_{i j}=\left(\sigma_{i j}-l^{2} \Delta \sigma_{i j}\right)$, which is solved with respect to local stresses $\sigma_{i j}$.

\section{II. ГРАДИЕНТНОЕ РЕШЕНИЕ ДЛЯ КОНЕЧНОЙ ТРЕЩИНЫ}

Let us consider a plane problem for an infinite strip loaded by stresses $\sigma$ in the direction of the axis OY and containing a finite crack of length $2 l,-l \leq x \leq l, y=0$. The classical solution to this problem is written in a complex-valued form through harmonic and biharmonic potentials $\phi(w)$ and $\psi(w), w=x+i y$

$$
\begin{aligned}
& \tau_{11}=\tau_{x x}=\sigma \operatorname{Re}(\phi+\psi), \tau_{22}=\tau_{y y}=\sigma \operatorname{Re}(\phi-\psi), \\
& \tau_{x y}=\sigma \operatorname{Im} \psi
\end{aligned}
$$

Here we must take into account that

$$
\begin{gathered}
\phi=\phi_{0}, \quad \psi=\psi_{0}, \phi_{0}=w /\left(w^{2} /-l^{2}\right)^{1 / 2}, \\
\psi_{0}=l^{2}(w-w) /\left[2\left(w^{2}-l^{2}\right)^{32}\right] .
\end{gathered}
$$

Let's construct a generalized solution that does not have singularities at the origin. To do this, we find regular solutions for the harmonic and biharmonic functions $\phi$ and $\psi$, that satisfy the equations $\phi-s^{2} \nabla^{2} \phi=\phi_{0}$, $\psi-s^{2} \nabla^{2} \psi=\psi_{0}$, compensating the features of particular solutions of the inhomogeneous Helmholtz equations (classical singular solutions) at singular points 
$x= \pm l, \quad y=0$ using radial factors [7] $\chi_{0}=e^{-r / s} w^{-1 / 2}$, $\chi_{1}=(r / s+1) e^{-r / s} w^{-3 / 2}$

$\chi_{2}=\left((r / s)^{2}+3(r / s+1)\right) e^{-r / s} w^{-5 / 2} \quad, \quad$ satisfying the

Helmholtz equation and having a set of the same features. As a result, we find

$$
\begin{aligned}
\phi & =w\left(w^{2}-l^{2}\right)^{-1 / 2}-(l / 2)^{1 / 2}\left(e_{1 / 2}^{+}+i e_{1 / 2}^{-}\right) \\
\psi & =\left(l^{2} / 2\right)(w-\bar{w}) /\left(w^{2}-l^{2}\right)^{-3 / 2}- \\
& -4(l / 2)^{1 / 2}\left(1-15 / 16(s / l)^{2}\right)\left(e_{1 / 2}^{+}-i e_{1 / 2}^{-}\right)+(4) \\
& +6 s^{2} l^{2} w\left(w^{2}-l^{2}\right)^{-5 / 2}+ \\
& +(3 / 16 \sqrt{2}) s^{2}(l)^{-1 / 2}\left(e_{3 / 2}^{+}+i e_{3 / 2}^{-}\right)- \\
& -(1 / 2 \sqrt{2}) s^{2}(l)^{1 / 2}\left(e_{5 / 2}^{+}+i e_{5 / 2}^{-}\right)
\end{aligned}
$$

where

$$
\begin{aligned}
& e_{1 / 2}^{+}=\exp \left(-r^{+} / s\right)(w-l)^{-1 / 2}, \quad e_{1 / 2}^{-}=\exp \left(-r^{-} / s\right)(w+l)^{-1 / 2} \\
& e_{3 / 2}^{ \pm}=\left(r^{+} / s+1\right)(w \mp l)^{3 / 2} \exp \left(-r^{ \pm} / s\right) \\
& e_{5 / 2}^{ \pm}=\left(\left(r^{ \pm} / s\right)^{2}+3\left(r^{ \pm} / s+1\right)\right)(w \mp l)^{5 / 2} \exp \left(-r^{ \pm} / s\right) \\
& r^{ \pm}=\sqrt{(w \mp l)(\bar{w} \mp l)}
\end{aligned}
$$

Nonsingular stresses are determined by potentials $\phi$ and $\psi$ by formulas (3),(4). For example, local stresses on the material axis (s) can be determined ( $\hat{x}=x / l$ and $\lambda=l / s)$ :

$$
\begin{aligned}
\sigma_{y y} & =(\sigma / 2)\left[\hat{x}\left(\left(\hat{x}^{2}-1\right)^{2}-6 \lambda^{-2}\right)\left(\hat{x}^{2}-1\right)^{-5 / 2}-\right. \\
& -(8 \sqrt{2})^{-1}\left(2+3 \lambda^{-1}+15 / 8 \lambda^{-2}\right)\left(\hat{e}_{1 / 2}^{+}+\hat{e}_{1 / 2}^{-}\right)- \\
& -3(8 \sqrt{2})^{-1}\left(\lambda^{-2}-4 \lambda^{-1}\right)\left(\hat{e}_{3 / 2}^{+}+\hat{e}_{3 / 2}^{-}\right)+ \\
& \left.+3 \lambda^{-2}(2 \sqrt{2})^{-1}\left(\hat{e}_{5 / 2}^{+}+\hat{e}_{5 / 2}^{-}\right)\right]
\end{aligned}
$$

where

$$
\hat{e}_{k / 2}^{ \pm}=(x \mp l)^{-1 / 2} \exp (-\lambda(\hat{x} \mp 1)), \quad k=1,3,5
$$

\section{GRADIENT SOLUTION FOR I INFINITE CRACKS}

To construct regular solutions for semi-infinite cracks in an isotropic strip, we use the Papkovich - Neuber representation to write down classical solutions in a convenient form through one complex-valued function $f=f_{x}+i f_{y}, f=f(w, \bar{w}), \quad \nabla^{2} f=0$ and for stress fields $\tau_{x x}=\operatorname{Re}(P+T), \quad \tau_{y y}=\operatorname{Re}(P-T), \quad \tau_{x y}=\operatorname{Im} T$, here $P, T$ are complex potentials. It is shown that classical solutions for displacements and stresses are written through one harmonic function:

$$
\begin{aligned}
& U=f / \mu-(1 / 4 \mu(1-v)) \partial(\bar{w} f+w \bar{f}) / \partial \bar{w} \\
& U=(1 / 2(1-v))(\partial f / \partial w+\partial \bar{f} / \partial \bar{w}) \\
& T=2(\partial f / \partial \bar{w})-(1 / 2(1-v)) \partial^{2}(\bar{w} f+w \bar{f}) / \partial \bar{w}^{2}
\end{aligned}
$$

Here $f(w, \bar{w})$ is the harmonic complex potential which for cracks of Mode I, Mode II and Mode III has the following form, respectively:

$$
\begin{gathered}
f=f_{I}=K_{I} \sqrt{2 / \pi}\left(w^{1 / 2}-\bar{w}^{1 / 2} /(5-8 v)\right)(1-v), \\
f=f_{I I}=-i K_{I I} \sqrt{2 / \pi}\left(w^{1 / 2}-3 \bar{w}^{1 / 2} /(5-8 v)\right)(1-v), \\
f=f_{I I I}=K_{I I I} \sqrt{2 / \pi} \operatorname{Im}\left(w^{1 / 2}\right)
\end{gathered}
$$

For the generalized gradient theory, local regular fields of stresses and displacements are found as solutions of the inhomogeneous Helmholtz equations:

$$
u-s^{2} \nabla^{2} u=U, \quad p-s^{2} \nabla^{2} p=P, \quad t-s^{2} \nabla^{2} t=T
$$

The right-hand sides of (7) contain singular complexvalued functions $U, P, T$. They are written for each mode of cracks through one harmonic function (6). Regular local solution i.e. potentials $p, t$, displacements $u=u_{x}+i u_{y}$ and stresses $\sigma_{x x}=\operatorname{Re}(p+t), \quad \sigma_{y y}=\operatorname{Re}(p-t), \quad \tau_{x y}=\operatorname{Im} t \quad$ is constructed using the radial factor method [7]. For Mode I crack, such a regular solution has the form

$$
\begin{aligned}
u & =K_{I} /(\mu \sqrt{2 \pi})\left[(3 / 2-2 v) w^{1 / 2}-\bar{w}^{1 / 2} / 4+\right. \\
& \left.+\left(s^{2} / 2\right)\left(1-(r / s)^{2} / 2+2 \hat{h}_{1}(r)\right) \bar{w}^{-3 / 2}\right] \\
p & =K_{I} / \sqrt{2 \pi}\left(1-\hat{h}_{0}(r)\right)\left(w^{-1 / 2}+\bar{w}^{-1 / 2}\right) / 2 \\
t & =-K_{I} /(4 \sqrt{2 \pi})\left[\left(1-\hat{h}_{0}(r)\right) w^{-1 / 2}+\right. \\
& \left.+6 s^{2}\left(1-(r / s)^{2} / 6-4 \hat{h}_{2}(r) / 3\right) \bar{w}^{-5 / 2}\right]
\end{aligned}
$$

where

$$
\begin{gathered}
\hat{h}_{0}(r)=e^{-r / s}, \quad \hat{h}_{1}(r)=-\frac{r / s+1}{2} e^{-r / s}, \\
\hat{h}_{2}(r)=\frac{(r / s)^{2}+3(r / s+1)}{4} e^{-r / s}
\end{gathered}
$$

Regular solutions are built for both Mode II cracks and Mode III cracks. For example, we have for a complex-valued displacement function for cracks of Mode III

$$
\begin{aligned}
& u=-i K_{I} /(\mu \sqrt{2 \pi})\left[(3 / 2-2 v) w^{1 / 2}-\right. \\
& \left.-3 \bar{w}^{1 / 2} / 4+\left(s^{2} / 2\right)\left(1+(r / s)^{2} / 2+2 \hat{h}_{1}(r)\right) \bar{w}^{-3 / 2}\right]
\end{aligned}
$$

and, accordingly, a regular solution for Mode III cracks

$$
\begin{aligned}
& u_{z}=\left(K_{I I} / \mu\right) \sqrt{2 r / \pi} \sin (\theta / 2) \\
& \sigma_{x z}=-K_{I I I} /(\sqrt{2 \pi r})\left(1-\hat{h}_{0}(r)\right) \sin (\theta / 2) \\
& \sigma_{y z}=K_{I I I} /(\sqrt{2 \pi r})\left(1-\hat{h}_{0}(r)\right) \cos (\theta / 2)
\end{aligned}
$$

\section{The concept of stress concentration}

Nonsingular solutions obtained using gradient models (4), (7) - (10) are the basis for implementing the concept of stress concentration in crack mechanics proposed in [8]. For example, for a Mode I crack, the stress expression $\sigma_{y y}$, $\hat{r}=r / l, \lambda=l / s$ calculated over the crack extension can be used as an estimate of strength. 


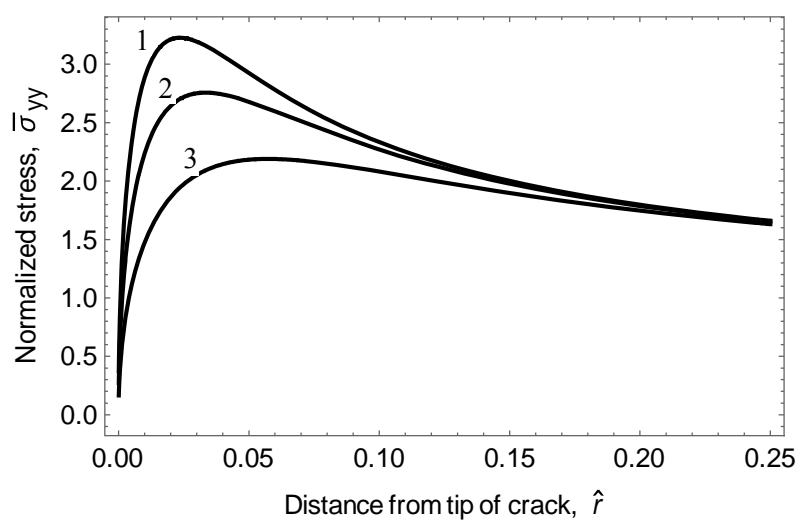

Fig. 1. Distribution of the normalized stress from normalized distance of tip of $\operatorname{crack}(1 .-\lambda=70,2-\lambda=50,3$.- $\lambda=30)$

The distribution $\sigma_{y y} / \sigma=\bar{\sigma}_{y y}(\hat{r} \lambda)$, is a positive bounded and continuous function of the parameter $\hat{r}$ over the entire determination interval $0<\hat{r}<\infty$, which becomes to zero at $\hat{r}=0$ typical for stress concentration. Strength can be estimated by the level of ultimate stresses $\sigma_{y y}$. The typical distribution of the nonsingular normalized stresses near tip of crack Mode I for different value of the normalized scale parameter $\lambda$ is shown on the Figl.

Instead of $\sigma^{m}=\sigma_{y y}^{\max }$ the magnitude of the stress intensity

$$
\sigma^{m}=\underbrace{\max }_{0<\hat{r}<\infty} \sigma_{i}=\underbrace{\max }_{0<\hat{r}<\infty} \sqrt{\sigma_{x x}^{2}+\sigma_{y y}^{2}-\sigma_{x x} \sigma_{y y}}
$$

can be used when it comes to plastic materials. The found regular stress solutions allow us to calculate the stress concentration coefficient $k_{\sigma}=\sigma^{m} / \sigma$, where $\sigma^{m}$ is the maximum value of stresses $\sigma_{y y}$ (or stress intensity $\sigma_{i}$ ). The calculated stress concentration coefficient depends on the scale parameter $\lambda$. The obtained calculated strength estimates are compared with experimental ones.

An experimental study was carried out on plates of aluminum alloy, brass and steel. A crack was defined as a side cut in a strip that was loaded according to a three-point bending test.

As an example, we briefly discuss the experiment and calculation for aluminum alloy plates. The theoretical curve of the proportionality limit constructed from the stress intensity is shown in Fig 2.

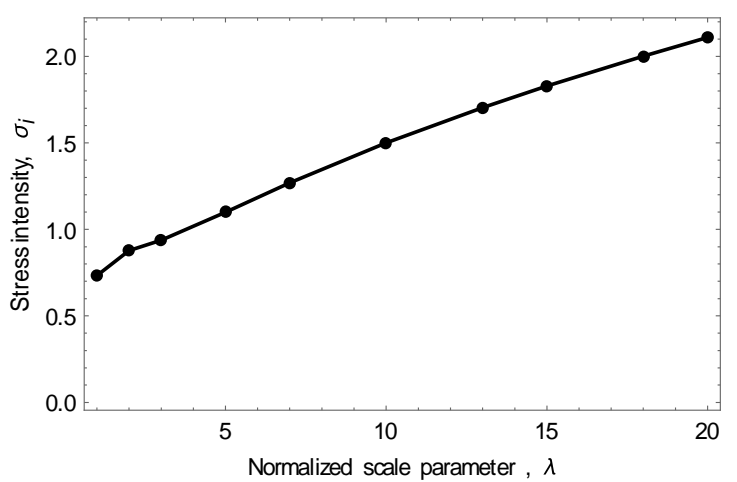

Fig. 2. Theoretical depenence of normalized stress intensity $\sigma_{i} / \sigma$ from scale parameter $\lambda$
For a plate with a crack $5 \mathrm{~mm}$ long, the experimentally established proportionality limit is $\sigma=65 \mathrm{MPa}$. Thus, the stress concentration coefficient $k_{\sigma}=\sigma_{u} / \sigma=1.154$ and according to Fig. 2 we have $\lambda=5.5 \mathrm{~mm}$ and then $s=l / \lambda=0.91$. Further, assuming that the value of $s=0.91$ $\mathrm{mm}$ is unchanged for the material under consideration, we give a forecast of the proportionality limit for the strip with different long cracks. For a plate with a crack of length $l=10 \mathrm{~mm}$ at $\mathrm{mm}$, we have $\lambda=11, k_{\sigma}=1.56$ and predict using Fig.2 ultimate stresses (proportionality limit) $\sigma=48.1 \mathrm{MPa}$. For $l=15 \mathrm{~mm}$, we get $\lambda=16.5, k_{\sigma}=1.93$ and $\sigma=38.9 \mathrm{MPa}$. Finally, for $l=20 \mathrm{~mm}$, we also find $\lambda=22, \quad k_{\sigma}=2.22$ and $\sigma=33.8 \mathrm{MPa}$. As a result, it turned out that these values of the ultimate stresses obtained theoretically coincide with experimental accuracy with high accuracy (the correlation coefficient is less than 6\%).

As a result, parameter $\lambda=l / s$ is determined and scale parameter $s$ is found, which, along with the tensile strength, is a fracture characteristic. The experiments were carried out both for brittle and plastic materials. It is shown that the parameter $s$ for a particular material is a constant value and, along with the tensile strength, can be considered as a fracture parameter, giving a high accuracy forecast for the strength of materials. Moreover, the concept of stress concentration made it possible to predict theoretically the effect of increasing the proportionality limit in the vicinity of the crack tip for plastic materials, which was also confirmed by experiment. It is noted that for a plastic material this parameter is approximately an order of magnitude larger than the value obtained above for a brittle material.

\section{ACKNOWLEDGMENT}

The authors acknowledge with thanks support from the Russian Science Foundation grant No. 19-01-00355. This work was carried out also with the partial support of Russian Government Foundation to the Institute of Applied Mechanics of RAS (no. AAAA-A19-119012290177-0).

\section{REFERENCES}

[1] A.Carpinteri, M. Paggi, "Asymptotic analysis in Linear Elasticity: From the pioneering studies by Wieghardt and Irwin until today," Eng. Fract. Mech., vol. 76, pp. 1771-1784, 2009.

[2] G.C. Sih, X.S.Tang, "Scaling of volume energy density function reflecting damage by singularities at macro-, meso- and microscopic level," Theor. Appl. Fract. Mech., vol. 43, pp. 211-231, 2005.

[3] M. Gutkin, E. Aifantis, "Dislocations in the theory of gradient elasticity," Scripta mater. vol. 40, no. 5, pp. 559-566, 1999.

[4] S. Lurie, P. Belov, "Gradient effects in fracture mechanics for nanostructured materials," Eng. Fract. Mech., vol.130, pp. 3-11, August 2014.

[5] V.Vasiliev, S. Lurie, "Nonlocal Solutions to Singular Problems of Mathematical Physics and Mechanics," Mech. Solids, vol.53, pp.135144, 2018.

[6] V. Vasiliev, S. Lurie, "Generalized theory of elasticity," Mech. Solids vol. 50, pp.379-388, 2015.

[7] S. Lurie, D. Volkov-Bogorodskiy, E. Moiseev, A. Kholomeeva, "Radial multipliers in solutions of the Helmholtz equations," Integral Transforms Special Funct. Vol. 30, pp. 254-263, 2019.

[8] V.Vasiliev, S. Lurie , V. Salov, "Estimation of the Strength of Plates with Cracks Based on the Maximum Stress Criterion in a ScaleDependent Generalized Theory of Elasticity". Physical Mesomechanics, vol 22, no. 6, pp. 456-46, 2019. 